\title{
Réalisme poétique chez Marie-Claire Blais
}

\author{
Isabelle Favre \\ University of Nevada, Reno
}

M arie-Claire Blais est aujourd'hui l'une des figures de proue de la littérature francophone nord-américaine. Son œuvre prolifique se distingue par le soin méticuleux qu'elle confère aux détails, ainsi qu'à une riche contextualisation narratologique. Le résultat de ce travail est l'engagement progressif du texte dans des réalités très spécifiques choisies par l'auteure; les choix de Blais répondent à un fort besoin de dire, de peindre, de faire vivre, aimer, souffrir, des personnages sortis tout droit de "la vraie vie."

Réalité et vérité forment un couple presque indissociable, que devient-il lorsqu'on le couche sur papier? La langue est composée de signes; s'il est vrai que le mot "chien" ne mordra jamais, il n'en demeure pas moins que le signe "Chien méchant" peut déclencher une peur bien réelle. Dans une perspective phénoménologique, la réalité du monde est celle que nous percevons; or l'écriture et la lecture participent d'un procédé qui non seulement passe mécaniquement par les sens, mais qui en outre possède la faculté de déclencher des perceptions plus fines comme l'expérience esthétique, ou plus fortes comme le sentiment de révolte. Dans l'essai qui suit, je me propose d'explorer comment l'expression poétique et l'inscription du réel se combinent et se complètent dans l'écriture de Blais; j'essaierai de montrer dans quelle mesure, et par quels procédés linguistiques, l'auteure n'hésite pas à se lancer dans l'exploration littéraire de réalités aussi diverses que marginales. Il me paraît nécessaire d'insister sur la diversité des sujets traités dans cette œuvre qui met parfois en scène des personnages homosexuels, tant est rampant le préjugé qui consiste à accuser toute manifestation ou -plus gravement encore- tout être homosexuel, d'égotisme ou d'obsession avec sa propre différence.

Ainsi dans Manuscrits de Pauline Archange, la narration dépeint le vécu d'une petite fille de la classe ouvrière montréalaise des années cinquante, pour ensuite passer avec Les Nuits de l'Underground, à une vision post-industrielle du milieu lesbien contemporain. Dans chacun des romans cités précédemment, l'auteure choisit un contexte socio-politique tout à fait concret, puis elle le façonne et le réalise patiemment en travaillant les ressources poétiques de la langue. Kristeva met l'accent sur l'aspect 
pulsionnel du langage poétique, qui dès la fin du XIX ème siècle se libère de contraintes conscientes telles que la métrique, laissant la voie ouverte à ce qu'elle appelle "le frayage des pulsions." Dans son analyse:

Le texte qui en résulte se donne alors comme un dispositif sémiotique bouleversant la normativité du langage de la communication: son lexique, sa syntaxe, ses rapports contextuels, ses instances subjectives. Or, toutes ces modifications ne font qu'enregistrer, en fait, un bouleversement profond dans le statut du sujet parlant. (Kristeva 612)

Les textes de Blais fournissent une excellente illustration de l'argument présenté ci-dessus. En effet, son écriture joue constamment avec les différents niveaux de langue; la présence du joual dans ses pages "bouleverse" véritablement non seulement le statut du sujet, mais également le statut de la langue elle-même. Le joual de Blais produit un effet de réel qui interpelle le lecteur/trice non pas au niveau du message, mais à un niveau plus direct, sonore et vivide, dans lequel les personnages signalent leur réalité physique à travers l'usage qu'ils font de la langue. En conséquence, les dialogues de l'auteure suggèrent le langage du corps des protagonistes de façon à ce que le lecteur soit à même de visualiser la personne qu'il "entend." Cette synesthésie littéraire est illustrée dans le passage suivant des Manucrits de Pauline Archange, où l'enfant remarque que chaque adulte possède un mode d'expression qui lui est propre, même lorsque le sujet abordé est pareil. Pauline imite donc leur expression, comme si elle désirait rendre ces adultes réels et leur donner substance, en présentant leur performance langagière dans le contexte d'une variation sur le même thème:

" T'as donc pas une graine de conscience" disait ma mère. "Ayez une conscience pure", "Soyez blanches comme des lis..." disait madame la Directrice. "J'vais te nettoyer ta sale conscience" disait le père de Jacob à son fils. (98-99)

La performance orale donne corps et substance à l'hypocrisie religieuse et à la violence domestique qui constituent, dans ce texte, la toile de fond du vécu de Pauline. De plus, la forme orale est utilisée en lieu et place du procédé descriptif pour informer la lectrice des réalités socio-culturelles dans lesquelles se placent la narration; ainsi le père de Pauline souligne-t-il le problème de surpopulation qu'à connu le Québec jusqu'à un passé relativement récent:

- On a pas de misères, on est pas trop mal, tu te rappelles donc pas ce que c'était quand j'étais garçon? On était dix-sept, et tout le monde a appris à travailler. (131) 
Kristeva souligne la composante subversive des usages de langue capables de surprendre le lecteur par leur originalité esthétique; cette modification de la normativité linguistique entraîne avec elle la mise en procès des rapports langue/classe sociale. Ce point théorique est activé de façon très directe par la mère de Pauline qui désire quitter le quartier "dur" (131) dans lequel se déroule la narration, pour aller vivre à Saint-Thomas-des-Rois "C'est une paroisse distinguée" dit-elle, " il y a des arbres et on parle mieux qu'ici" (131). La distinction et le raffinement sont associés à un niveau de langue supérieur, lieu de désir de la mère, alors qu'elle évolue linguistiquement - et par extension physiquement - dans une "dure réalité." Il est opportun d'insister sur la difficulté stylistique que représente la retranscription de l'oralité. Cette forme littéraire sollicite en effet une grande finesse d'écoute ainsi qu'un choix lexical comparable à l'entreprise poétique, dans le sens où toutes deux se trouvent constamment menacées de surcharge et de déséquilibres linguistiques. Barthes articule éloquemment la spécificité de ce qu'il appelle l'écriture à haute voix:

Son objectif n'est pas la clarté des messages, le théâtre des émotions; ce qu'elle cherche (dans une perspective de jouissance), ce sont les incidents pulsionnels, c'est le langage tapissé de peau, un texte où l'on puisse entendre le grain du gosier, la patine des consonnes, la volupté des voyelles, toute une stéréophonie de la chair profonde. ( Barthes 105)

Si les corps qui émergent des Manuscrits sont souvent des corps de souffrance, ils possèdent également et peut-être en raison de leur souffrance même, une faculté de jouissance hors du commun qui entraîne la narration dans un monde pulsionnel intense et surprenant qui participe à la réévaluation des hiérarchies socio-linguistiques. C'est ainsi que les pauvres conditions de vie de Pauline l'exposent à toutes sortes d'expériences heureuses et malheureuses, qui développent chez cet enfant une capacité émotionnelle riche et intense, en comparaison de laquelle la vie d'une petite fille "normale" paraît tout à fait ennuyeuse et infantile, dans le sens péjoratif du terme. Cette réévaluation des classes sociales en terme de richesse intérieure est un projet cher à l'entreprise littéraire de l'auteure.

Les personnages de l'auteure québécoise prennent certes la parole, mais aussi et surtout, ils pensent. Une grande partie des textes de Blais se situent dans une réalité souvent mise en cause en temps que telle, parce que prenant place dans l'espace intangible des réflexions intérieures. Monde d'abstraction qui partage avec l'univers du récit un statut marginal, un peu hors jeu, hors monde. Toutefois, comme l'observe Genette au sujet d'Oedipe, le récit n'est certes pas dépourvu de pouvoir direct sur le déroulement de l'événement réel à venir :

Ce n'est pas une prophétie qui se réalise, c'est un piège en forme de récit, 
et qui "prend". Oui, puissance (et ruse) du récit. Il en est qui font vivre (Schéhérazade), il en est qui tuent." ( Genette 251)

En effet, la réalisation de l'être au monde collectif passe par de multiples récits qui participent à la mise en place chez l'individu, des structures socio- culturelles. Les éléments issus du réel tangible sont assemblés, associés, orientés en fonction de modèles représentatifs tirés des nombreux récits qui parcourent incessamment les ondes, les livres ou les lèvres. Cette puissance du récit dont parle Genette tient aussi au fait qu'il fonctionne comme agent cohésif dans un univers de signes aussi abondant que chaotique. La perception du réel passe par la catégorisation des données et la répétition de comportements concrets découlant de cette classification. Si l'impact de la chose racontée est indéniable, qu'en est-il des histoires que l'on ne raconte pas? Qu'advient-il des réalités dont le signifiant n'est pas activé? Le vrai pouvoir de vie ou du mort du récit pourrait bien se trouver ailleurs, en dehors, dans le non-récit qui provoque le vrai hors jeu, le hors catégorie, et la non appartenance. Dans cette perspective, on ne peut que remarquer l'absence évidente de textes et contextes lesbiens au sein du corpus littéraire français/francophone. Marie-Claire Blais est donc une des rares auteures francophones qui s'engage à briser un silence littéraire aussi béant que significatif.

Selon Sartre, l'écrivain est un personnage qui a le choix d'intégrer de nouveaux éléments ou de nouvelles catégories dans la grille du récit: “Le prosateur est un homme qui a choisi un certain mode d'action secondaire qu'on pourrait nommer l'action par dévoilement." (Sartre 30) Blais utilise effectivement abondamment le discours indirect pour dévoiler les pensées de ses personnages. L'utilisation du verbe "dévoiler" dans un contexte proche de sexualités non sanctionnées, s'associe très rapidement à des images de révélations, de confessions, portant sur le dévoilement d'actions cachées. Il n'en est pourtant rien dans ces textes: nulle trace de "sorties du placard," nul personnage "ambigu," aucune "grave confusion identitaire" ne concourt à l'élaboration du récit. Le lesbianisme des personnages découle de l'un des éléments constitutifs de la réalité elle-même: c'est une donnée, un fait que l'on n'a pas loisir de remettre en question en tant que tel. Toutefois, les personnages clés de Blais interrogent et s'interrogent incessamment, mais leur questionnement se situe bien audelà de la simple reconnaissance de leurs désirs. Dans Les Nuits de l'Underground par exemple, Geneviève est une artiste qui poursuit une longue quête dans laquelle elle tente de conjuguer deux réalités: sa vie de sculpteur, et sa vie amoureuse:

Elle n'aimait pas Lali, elle aimait en elle la beauté, la perfection de l'art. Mais ce qui la désemparait, c'était de comprendre que l'art est partout vivant et charnel, que ce qu'elle avait vu au loin et sans danger, dans la confiance des musées, vivait et frémissait tout près d'elle, dans sa vie même, cette œuvre vivante, c'était Lali [...] (22-23) 
La perspective esthétique du personnage se rapproche de la modernité et du dandysme. La poétisation de Lali passe par la réification : le regard de Geneviève la transforme en objet, ce qui lui confère une certaine qualité de perfection que l'on ne peut trouver que dans la beauté inanimée; cette beauté parfaite que l'on pourrait qualifier d' inhumaine, constitue l'un des topoi majeurs du dandysme. Les différentes descriptions de Lali évoquent un sujet dandy revisité dans une perspective lesbienne post-industrielle. Lali est en effet grande et mince, toutefois contrairement aux dandys, sa maigreur dégage également une impression de force. Tout comme eux, ses vêtements déclenchent le commentaire descriptif, mais chez elle l'élégance est moins importante que le style: son manteau d'hiver n'est pas coupé, il est "long et droit,"(16) ses mains sont enfouies dans des gants d'hommes certainement trop grands qui ne sont ni en soie ni en daim, mais doublés de mouton. Comme tout dandy, le comportement social de Lali intrigue, surprend. Au bar de l'Underground, bien que familière de l'endroit et de ses clientes, elle s'assied toute seule devant une bière et ne parle à personne ce qui -comme le souhaitaient les dandy- suscite diverses productions discursives dont elle est le sujet. Dans ce roman, l'attitude distante et mystérieuse de Lali au bar, entraîne la narration à développer la perception poétique du personnage. Lali se dessine lentement dans le texte, elle prend peu à peu forme à travers les mots d'une femme sculpteur: "Geneviève croyait admirer un tableau de Van Eyck, n'osant pas convoiter un visage qui incarnait pour elle une lointaine et mélancolique spiritualité[...]" (12)

Geneviève est le personnage qui, en raison de sa passion pour les arts plastiques, se situe près des "choses" en tant qu'expressions artistiques; elle travaille la matière et produit une ouvre qui se présente dans toute sa réalité objective. L'intérêt narratologique d'un tel personnage réside dans le fait qu'il donne l'occasion au texte de traduire en signes l'objet porteur de beauté; cette mise en symbole de l'expression artistique donne lieu à l' expression poétique qui elle, ne peut avoir lieu que par et dans la langue. Dans Qu'est-ce que la littérature? Sartre associe les arts plastiques à la "chose" et à la poésie, car ils procèdent tous deux d'une réalité immédiate:

[...] l'ambiguiité du signe implique qu'on puisse à son gré le traverser comme une vitre et poursuivre à travers lui la chose signifiée ou tourner son regard vers sa réalité et le considérer comme objet. (Sartre 18)

Les œuvres de Blais conjuguent "l'ambiguïté du signe" mentionnée plus haut: ses mots deviennent parfois choses, ils ne renvoient qu'à eux-mêmes et au plaisir de lire/ou écrire. Mais le texte continue et la prose de l'auteure - ponctuée de moments poétiques - poursuit effectivement la, ou les choses signifiées. Blais engage ses textes dans des réalité qui se situent au-delà du signe et du livre; il est évident par exemple qu'elle cherche à faire une peinture réaliste de l'être au mondes lesbien. Pour cela, elle 
établit un mouvement textuel alterné entre la réalité intérieure de ses personnages, constituée de réflexions souvent intenses et difficiles, et le monde extérieur qui se manifeste à travers l'oralité. A l'Underground, la saveur du joual persiste mais cette fois, il a une fonction cohésive; contrairement à sa présence rude et même rustre dans la "vraie" famille de Pauline Archange, le joual que l'on entend à l'Underground crée une famille dépourvue de réalité biologique, mais unie par de forts liens d'amitié.

Les dialogues qui prennent place dans le bar établissent un contact direct entre les personnages, plaçant ainsi un écran momentané entre la lectrice et les personnages du récit. L'intimité qui s'était établie lorsque le texte faisait partager les pensées du personnage au lecteur, s'interrompt soudain. Les personnages s'adressent subitement les uns aux autres et le lecteur passe du statut de confident, à celui de spectateur. Ces mouvements discursifs alternés de rapprochement/distanciation, rythment d'une part les pages du texte et déclenchent également ce que les anglophones appellent "a reality check." En effet, les réflexions exigeantes et ardues de Geneviève au sujet des rapports entre son désir amoureux et son amour de l'art, sont interrompues par l'irruption sur la page de commentaires beaucoup plus "terre à terre," portant eux aussi sur le personnage-dandy du texte:

Elle s'appelle Lali Dorman, elle vient de quelque part en Europe, L'Autriche, je pense, elle est fatiguée ce soir et ne veut parler à personne[...] Y a bien six ou sept ans que je la vois chez nous[...] Je l'ai aperçue dans un autre bar, un soir de bataille, elle était seule comme ce soir[...] un peu triste, c'est normal[...] pourquoi s'amuser quand on feele pas pour ça? Elle est médecin je pense[...] c'est pas une vie drôle, toujours à l'hôpital comme moi, on en voit de toutes les couleurs! (13)

Ce fragment dialogique fournit au lecteur une perspective différente du même personnage: la vision poétisée de Lali semble à nouveau "sortir du musée" et se mue en une description plus précise qui intègre Lali dans le monde, plutôt que de l'extraire de celui-ci. De plus, le récit confirmera le rôle important que joue la profession dans la vie du personnage; contrairement aux dandys, Lali s'engage dans la société. Les nombreuses touches de réalisme social que l'on trouve dans les romans de Blais participent à un dessein qui vise à soulever une problématique qui devrait se poursuivre bien après la lecture du livre. Ses textes sont en effet soigneusement construits de façon à éclairer littérairement divers sujets en relation directe avec les débats de politique sexuelle. Les personnages de Blais exemplifient clairement la dissémination gay: elle les situe dans différents secteurs professionnels, à tous les niveaux économiques, et dans des postes à responsabilités variées. Leurs échanges à l'Underground confirment l'image d'une communauté diversifiée et tout à fait saine, qualificatif révélateur d'une certaine résistance active de l'auteure face à l'homophobie décrite plus loin. Si les textes de Blais ne peuvent être réduits à une simple réponse 
littéraire aux accusations anti-gay, ils familiarisent indéniablement ses lecteurs à un milieu réel, vivant, qui reste souvent figé dans des représentations partisanes. Une vaste campagne politique en provenance de l'extrême droite religieuse et laïque, s'applique à associer la communauté homosexuelle entière à la maladie et à la contamination. Cette perception haineuse autant qu'erronées est vigoureusement dénoncée par Linda Singer:

In light of the strategic value of epidemics, it is also important to point out that in contempory context, the primary medium for circulating the hegemony of epidemic has not been a medical discourse, but an explicitely political and ideological one. (Singer 118)

A nouveau, le pouvoir des mots se manifeste, de même que leur impact réel dans la société; le mot «épidémie» est tout à fait capable d'une part, d'éveiller des phobies endormies, et d'autres part d'en créer de nouvelles. Les propos homophobes des décennies précédentes se réclamaient directement de la morale en utilisant les champs sémantiques de la perversion, de la nature, et du diable. Avec l'épidémie, Satan se retrouve muté dans le département de l'hygiène et de la santé publique... Or, comme le signale Singer, le concept d'épidémie associé au Sida ne provient pas du discours médical; l'image épidémique vise à véhiculer l'idée d'une prolifération incontrôlée d'individus malades dont la présence constitue une menace pour la collectivité.

La peur, l'anxiété et la phobie participent largement des mécanismes de l'inconscient; les propos générés par ce genre d'émotions tendent à l'auto-justification en utilisant un discours référentiel parallèle qui se veut "rationnel" ou "scientifique." Lorsque l'objet déclencheur de phobie prend corps, apparaît et s'anime, il perd aussitôt une grande partie de sa puissance irrationnelle. Les nombreux personnages lesbiens qui apparaissent dans Les Nuits de l'Underground contribuent ainsi à déréaliser tout un système de représentation homophobe, pour le remplacer par les Tableaux d'une Exposition mouvante dont la musique, souhaitons-le, contribue aussi à adoucir les mours.

\section{Ouvrages cités}

Barthes, Roland Le plaisir du texte. Paris: Seuil, 1973.

Blais, Marie-Claire Manuscrits de Pauline Archange. Montréal: Stanké, 1981, 1968.

. Les nuits de l'Underground. Montréal: Boréal, 1990, 1978.

Genette, Gérard Figures II. Paris: Seuil, 1969.

Kristeva, Julia La Révolution du langage poétique. Paris: Seuil, 1974.

Sartre, Jean-Paul Qu'est-ce que la littérature? Paris: Gallimard, 1948.

Singer, Linda Erotic Welfare: sexual theory and politics in the age of epidemy.

Edited by Judith Butler. New York: Routeledge, 1992. 